\title{
Depressive Symptoms at Discharge from Rehabilitation Predict Future Cardiovascular-Related Hospitalizations
}

\author{
Fiorenza Angela Meyer ${ }^{\mathrm{a}}$ Eva Hugentobler ${ }^{\mathrm{a}}$ Stefanie Stauber ${ }^{\mathrm{c}}$ \\ Matthias Wilhelm ${ }^{d}$ Hansjörg Znoj ${ }^{b}$ Roland von Känel ${ }^{a, e, f}$ \\ aPsychosomatic Medicine Research Group and ${ }^{b}$ Institute of Psychology, Division of Clinical Psychology \\ and Psychotherapy, University of Bern, ${ }^{\mathrm{C}}$ Heart Failure and Transplantation and ${ }^{\mathrm{d} C a r d i o v a s c u l a r}$ Prevention, \\ Rehabilitation and Sports Medicine, Department of Cardiology, and eDepartment of Neurology, Inselspital, \\ Bern University Hospital and University of Bern, Bern, and fDepartment of Psychosomatic Medicine, \\ Clinic Barmelweid, Barmelweid, Switzerland
}

\author{
Key Words \\ Cardiac rehabilitation - Depression - Mortality · Prospective \\ longitudinal study
}

\begin{abstract}
Objectives: Depression is associated with poor prognosis in patients with cardiovascular disease (CVD). We hypothesized that depressive symptoms at discharge from a cardiac rehabilitation program are associated with an increased risk of future CVD-related hospitalizations. Methods: We examined $486 \mathrm{CVD}$ patients (mean age $=59.8 \pm 11.2$ ) who enrolled in a comprehensive 3-month rehabilitation program and completed the depression subscale of the Hospital Anxiety and Depression Scale (HADS-D). At follow-up we evaluated the predictive value of depressive symptoms for CVD-related hospitalizations, controlling for sociodemographic factors, cardiovascular risk factors, and disease severity. Results: During a mean follow-up of $41.5 \pm 15.6$ months, 63 patients experienced a CVD-related hospitalization. The percentage of depressive patients (HADS-D $\geq 8$ ) decreased from $16.9 \%$ at rehabilitation entry to $10.7 \%$ at discharge. Depressive symptoms at discharge from rehabilitation were a significant predictor of outcome (HR 1.32, 95\% Cl 1.09-1.60; $\mathrm{p}=$
\end{abstract}

0.004). Patients with clinically relevant depressive symptoms at discharge had a 2.5 -fold increased relative risk of poor cardiac prognosis compared to patients without clinically relevant depressive symptoms independently of other prognostic variables. Conclusion: In patients with CVD, depressive symptoms at discharge from rehabilitation indicated a poor cardiac prognosis.

(c) 2015 S. Karger AG, Basel

\section{Introduction}

Cardiovascular disease (CVD) is the major cause of death in adults in developed countries [1]. Even if the patient survives the first CVD event, there is a $43 \%$ chance of experiencing a recurrent CVD-related event in the first year [2]. Psychosocial risk factors increase the risk of CVD independently of traditional cardiovascular risk factors (CVRFs) $[3,4]$. In particular, clinically relevant levels of depressive symptoms are prevalent among patients with CVD; for instance, $20 \%$ of myocardial infarction patients have major depression and another $20 \%$ have minor depression [5]. Depression does not only increase the risk of the first CVD event, but it may also act as a barrier to treat-

Fiorenza Angela Meyer, MS

Department of General Internal Medicine, Division of Psychosomatic Medicine University Hospital, Inselspital

$\mathrm{CH}-3010$ Bern (Switzerland)

E-Mail fiorenza.meyer@gmx.ch 
ment efforts and to improving lifestyle [3]. Meta-analyses emphasize the unfavorable impact of depression on the prognosis of patients with coronary heart disease (CHD) $[6,7]$. However, conclusions are limited by the heterogeneity of the reviewed studies. Literature also confirmed that cardiac rehabilitation programs reduce subsequent CHD events, the total mortality risk and depression by $40-50 \%[8,9]$. However, few studies have evaluated the predictive value of depression during rehabilitation in relation to subsequent CVD-related hospitalizations.

To our knowledge, no other studies to date have analyzed whether depression at discharge from cardiac rehabilitation is prospectively associated with an increased risk of subsequent CVD-related hospitalizations in a sample of patients with different primary diagnoses of CVD. We decided to focus on depressive symptoms at discharge from rehabilitation because we strived to exclude patients with spontaneously remitting depressive symptoms and/ or those with a short-term depressive adjustment disorder in response to a CVD event [10]. Additionally, previous studies have shown that CHD patients whose depression scores do not improve during cardiac rehabilitation or psychotherapy are at a higher mortality risk compared to patients who respond to treatment $[9,11]$.

We performed a prospective study with patients who had participated in a cardiac rehabilitation program and examined their depression scores at discharge from the program with respect to future CVD-related hospitalizations. We hypothesized that depression is an independent predictor of CVD-related hospitalizations after controlling for demographic variables, traditional CVRFs and disease severity.

\section{Methods}

\section{Participants and Design}

All study patients participated in a comprehensive outpatient cardiac rehabilitation program at the Bern University Hospital, Switzerland, and were enrolled between January 2004 and December 2010. A detailed overview of the rehabilitation program has previously been published [12]. We included patients with a diagnosis of CVD (i.e., CHD, chronic heart failure and aortic valve surgery) who filled out a set of questionnaires during the first week and at discharge of an 8- to 12-week cardiac rehabilitation program. All patients provided written informed consent. The local ethics committee approved the study protocol. Demographic and medical data were obtained from hospital charts and through physical examination at the beginning of rehabilitation. The flowchart (fig. 1) illustrates the recruitment process for the patients. For the analysis we selected 486 patients from a total of 777 eligible patients. The group of patients who dropped out for reasons which are detailed in figure 1 did not significantly differ in terms of sex, age and educational background from the final study sample.

Depression and Cardiac Rehabilitation

Outcome

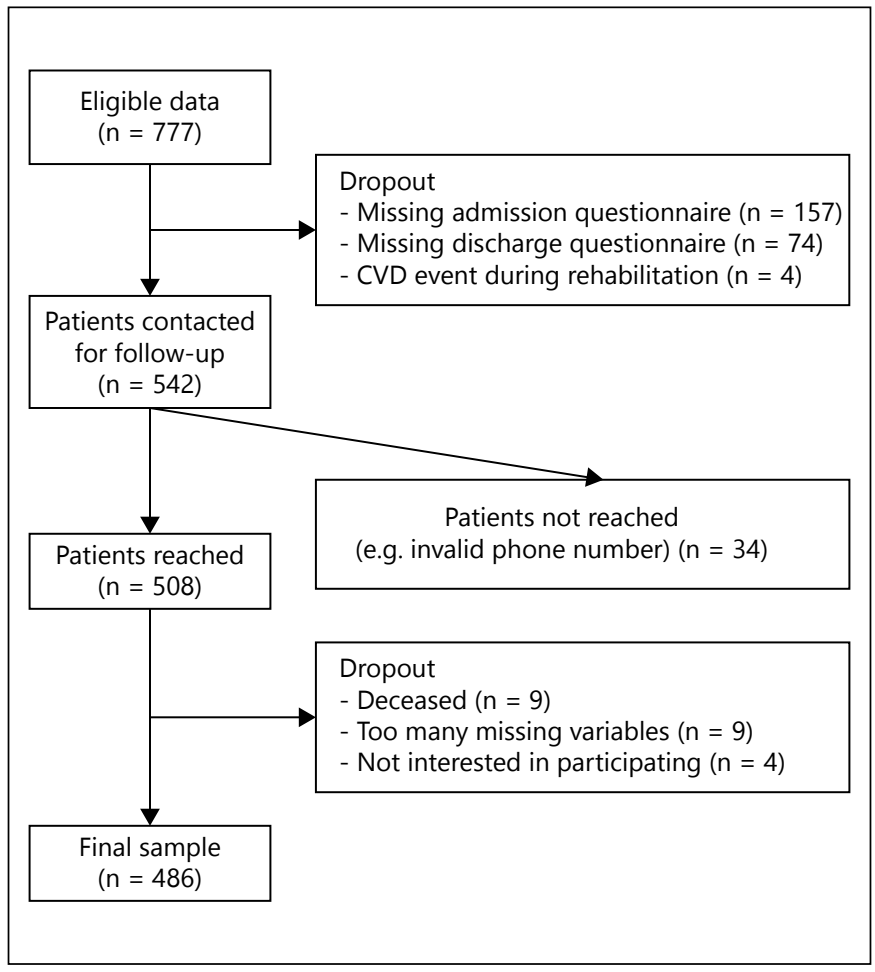

Fig. 1. Flowchart of patients selected for analysis.

Assessment of the Main Outcome Measure and Covariates

Depression was measured with the validated German version of the Hospital Anxiety and Depression Scale (HADS-D), which comprises seven items to assess the severity of depressive symptoms [13]. Patients have to rate on a 4-point Likert scale the symptom frequency (range from $0=$ 'mostly' to $3=$ 'not at all') with reference to the last week, yielding a total depressive symptom score ranging from 0 to 21 . In the present study a cutoff value of 8 points on the HADS-D scale was used to categorize the patients into two groups with high (score $\geq 8$ ) versus low (score $<8$ ) depressive symptom scores. We selected this cutoff value because it has previously been shown to optimally identify cases of depression with a mean sensitivity of 0.83 and a mean specificity of 0.79 [14].

We obtained information about sex, age, educational background and disease severity in the first week of the rehabilitation program. Traditional CVRFs were assessed while taking a medical history at the beginning and at discharge of the rehabilitation program. Body mass index was calculated on the basis of measured weight and height $\left(\mathrm{kg} / \mathrm{m}^{2}\right)$. A body mass index $\geq 30$ indicates obesity and was defined as a CVRF. Further CVRFs were diabetes, hypercholesterolemia, current smoking, a positive family history of CHD, and hypertension.

\section{Assessment at Follow-Up}

The follow-up period was defined as the time period between the discharge from the rehabilitation program and the semistructured telephone interview. The purpose of the telephone interview was to assess whether the patient has been rehospitalized due to a CVD-related event since discharge from the rehabilitation pro- 
gram. If a patient confirmed to have suffered a subsequent CVDrelated event, his or her primary care physician was contacted by phone and asked to send in a copy of the medical records for verification of the event. A total of 25 diagnostic categories and interventions were considered as subsequent CVD-related events.

\section{Statistical Analysis}

Data were analyzed using the SPSS 21.0 statistical software package (SPSS Inc., Chicago, Ill., USA) with a significance level of $\mathrm{p}<0.05$ (two-tailed). For the imputation of missing variables $(<5 \%)$ we applied the expectation maximum algorithm [15]. The Kolmogorov-Smirnoff test indicated a skewed distribution towards small values. However, no normal distribution was pursued because skewed predictors do not have an impact on regressiontype models [16]. Group differences in variables between patients with high versus low depressive symptom scores were calculated with the Mann-Whitney test.

For the main analysis we ran Cox proportional hazard models in order to define the predictive value of continuously and dichotomously scaled HADS-D scores for the relative risk of the endpoint hospitalizations due to a CVD-related event during followup. In the analysis with continuous HADS-D scores, the relative risk describes the predictive risk of scoring one point higher on the depression scale. A one-point interval is unlikely to be clinically relevant. Therefore, we expressed the change in the hazard ratio for a three-point higher level on the depression scale, marking transitions between mild, moderate and severe depression. Specifically, depression scores can be divided into four ranges of mood severity [17]. Scores 0-7 indicate no depression, 8-10 mild depression, 11-14 moderate depression and scores above 14 represent severe depression. We calculated the three-point interval by dividing the continuously scaled depression score by three and used the obtained measure in our analysis. Control variables for the model were defined a priori. As previously suggested, to prevent model overfitting given the number of CVD-related events, we performed data collapsing for CVRFs, whereby we assigned one point each for obesity, diabetes, hypercholesterolemia, current smoking, positive family history, and hypertension [16]. For the analysis, these points were added up to form a total CVRF score ranging from 0 to 6 . The final model was adjusted for sex, age, educational background, the CVRF score obtained at discharge from rehabilitation, and disease severity (i.e., the number of diseased vessels).

We performed two supplemental analyses. We calculated a complementary analysis adding each individual CVRF separately to the model because the collapsing of the traditional CVRFs into one sum score might obscure the predictive value of individual CVRFs. For a sensitivity analysis we excluded patients who suffered from endocarditis, pericarditis or pulmonary embolism as an outcome because these are not traditionally considered CVD events.

\section{Results}

\section{Patient Characteristics}

Except depressive symptom scores, there were no relevant changes in patient characteristics between rehabilitation entry and discharge, as best medical/cardiology treatment was already initiated in the acute care setting (data not shown in detail). Depression symptom scores significantly dropped from rehabilitation entry to discharge from rehabilitation $(4.2 \pm 3.5$, range $0-17$, vs. $3.3 \pm$ 3.3 , range $0-20, p<0.001)$. High depressive symptom scores (i.e., HADS-D scores $\geq 8$ ) were found in $16.9 \%$ of patients at rehabilitation entry and in $10.7 \%$ of patients at discharge from rehabilitation. Table 1 shows the characteristics of the total sample of patients at discharge from rehabilitation and for the two groups scoring high versus low in depressive symptoms.

\section{Cardiovascular Events during Follow-Up}

On average the follow-up telephone interview was conducted $41.5 \pm 13.6$ months (range 12-80) after discharge from rehabilitation. Of the 486 patients, 63 (12.9\%) experienced a CVD-related hospitalization during the follow-up period from which 5 patients had died (7.9\%). The CVD-related event occurred, on average, $20.9 \pm 16.0$ months (range 0.5-67) after discharge from rehabilitation. Sixteen different CVD-related events resulting in hospitalization were registered; these were elective intracoronary stenting $(42.8 \%)$, nonelective intracoronary stenting $(11.1 \%)$, cardiac arrhythmias $(6.3 \%)$, cerebrovascular insult $(6.3 \%)$, sudden cardiac arrest $(6.3 \%)$, pacemaker implantation $(4.7 \%)$, nonelective coronary angiography without stenting (3.2\%), chronic heart failure (3.2\%), pulmonary embolism (3.2\%), endocarditis (3.2\%), coronary artery bypass surgery (1.6\%), hypertensive crisis (1.6\%), aneurysm (1.6\%), Takotsubo cardiomyopathy (1.6\%), pericarditis $(1.6 \%)$, conduit change $(1.6 \%)$ and cardiac mortality (7.9\%).

\section{Cox Regression Model}

Depressive symptoms at discharge from rehabilitation were a significant predictor of future CVD-related events (HR 1.32, 95\% CI 1.09-1.60; $\mathrm{p}=0.004$ ). Thus, patients scoring three points higher on the depression scale had a $32 \%$ higher risk of experiencing a subsequent CVD-related hospitalization. The covariates sex, age, education, CVRF score and disease severity at discharge from rehabilitation did not emerge as independent predictors.

We ran a complementary analysis to possibly identify a predictive value of individual CVRFs that might have been obscured by collapsing all CVRFs across one sum score. This analysis showed that none of the CVRFs (i.e., diabetes, positive family history, hypertension, hypercholesterolemia, adiposity, current smoking) were independently predictive for subsequent CVD-related hospitalizations.

Performing a sensitivity analysis, we excluded 5 patients who had experienced pericarditis, endocarditis, or 
Table 1. Patient characteristics by depression level on the HADS at discharge (cutoff $\leq 8$ )

\begin{tabular}{|c|c|c|c|c|}
\hline Variable & $\begin{array}{l}\text { Total sample } \\
(\mathrm{n}=486)\end{array}$ & $\begin{array}{l}\text { High HADS score } \\
(\mathrm{n}=52)\end{array}$ & $\begin{array}{l}\text { Low HADS score } \\
(\mathrm{n}=434)\end{array}$ & $\mathrm{p}$ value \\
\hline Age (mean $\pm S D)$, years & $59.8 \pm 11.2$ & $57.4 \pm 11.4$ & $60.2 \pm 11.3$ & 0.051 \\
\hline Male gender, $\%$ & 78.0 & 75.0 & 78.3 & 0.583 \\
\hline Highest level of education, \% & & & & 0.944 \\
\hline Primary school & 5.5 & 9.6 & 5.1 & \\
\hline Vocational training & 68.3 & 61.5 & 69.1 & \\
\hline College or university & 26.1 & 28.8 & 25.8 & \\
\hline Diabetes, $\%$ & 12.6 & 17.3 & 12.0 & 0.274 \\
\hline Hypercholesterolemia, \% & 65.2 & 71.2 & 64.5 & 0.343 \\
\hline Smoking, \% & 40.3 & 46.2 & 39.6 & 0.365 \\
\hline Hypertension, \% & 61.1 & 61.5 & 61.1 & 0.947 \\
\hline Positive family history, $\%$ & 30.9 & 21.2 & 32.0 & 0.109 \\
\hline Obesity, \% & 18.7 & 21.2 & 18.4 & 0.635 \\
\hline Vessel disease, \% & & & & 0.583 \\
\hline 0 -vessel disease & 16.5 & 13.5 & 16.8 & \\
\hline 1-vessel disease & 33.3 & 34.6 & 33.2 & \\
\hline 2-vessel disease & 20.8 & 19.2 & 21.0 & \\
\hline 3-vessel disease & 29.2 & 32.7 & 28.8 & \\
\hline Aspirin, \% & 86.4 & 86.5 & 86.4 & 0.979 \\
\hline Beta-blockers, \% & 82.5 & 86.5 & 82.0 & 0.419 \\
\hline Statins, \% & 84.2 & 78.8 & 84.8 & 0.268 \\
\hline Diuretics, \% & 18.9 & 28.8 & 17.7 & 0.054 \\
\hline Angiotensin-converting enzyme inhibitor, \% & 61.9 & 61.5 & 62.0 & 0.950 \\
\hline Plavix, \% & 56.4 & 57.7 & 56.2 & 0.840 \\
\hline Depression score (mean $\pm \mathrm{SD})$ & $3.3 \pm 3.3$ & $10.5 \pm 2.4$ & $2.4 \pm 2.1$ & $<0.001$ \\
\hline
\end{tabular}

pulmonary embolism after discharge from rehabilitation as these outcomes are not traditionally considered CVD events. In this sensitivity analysis depressive symptoms at discharge from rehabilitation maintained their significant predictive value for CVD-related hospitalizations (HR 1.40, 95\% CI 1.15-1.69; $\mathrm{p}=0.001$ ).

In a final analysis we used the cutoff for clinically relevant levels of depressive symptoms as the predicting variable of interest. Individuals were grouped into patients with high depressive symptom scores (HADS-D score $\geq 8$ ) versus those with low depressive symptom scores (HADSD score $<8)$. Patients with high depressive symptom scores at discharge had a 2.5-fold increased relative risk of experiencing a CVD-related hospitalization (HR 2.57, 95\% CI 1.42-4.63; $\mathrm{p}=0.002$ ). The model was adjusted for sex, age, education, CVRF score, and disease severity.

\section{Discussion}

We found that depression scores during a comprehensive outpatient cardiac rehabilitation program predicted future CVD-related hospitalizations over a mean follow-

Depression and Cardiac Rehabilitation Outcome up period of almost 3.5 years. This association was independent of other potentially important predictors of poor prognosis in patients with CVD. Our results show that in a sample of 486 patients, $82(16.8 \%)$ patients scored high on the depression score at the beginning of rehabilitation, a number that concurs with previous research $[9,18]$. Upon completion of rehabilitation, only $10.7 \%$ of the patients suffered from a clinically relevant depression. By trend, patients in our study who had high levels of depressive symptoms were younger than those with low levels of depressive symptoms, concurring with findings by Lavie and Milani [19]. Younger CVD patients might be prone to depression because CVD manifests less frequently at a young age, whereas elderly patients might rather perceive CVD as part of normal ageing. Although not assessed in our study, younger patients might also have worse general health and health behaviors, including more sedentary behavior and unhealthy nutrition, both potentially increasing the level of depressive symptoms but also increasing the risk of premature CVD.

Depression was independently predictive of CVD-related hospitalizations in patients after cardiac rehabilitation. The continuous depression score demonstrated a 
dose-response relationship between the severity of depressive symptoms and poor outcome. Also Lespérance et al. [20] showed that the overall level of depressive symptoms predicted cardiac mortality in patients with CHD after a 5-year follow-up. Our findings that depressive symptom scores even below a clinically relevant cutoff are adversely associated with the prognosis of patients with CVD concur with results from two meta-analyses. According to Barth et al. [6], depressed CHD patients are at a twofold risk of dying in the subsequent 2 years. The investigation of 22 studies by van Melle et al. [7] came to similar conclusions. Most of the previous studies collected fatal endpoints but less so hospitalization data in relation to the predictive value of depression. Moreover, depression was measured solely after hospital admission and not in the context of a cardiac rehabilitation program. Therefore, our study design differs in several points from the bulk of prognostic studies in depressed cardiac patients and provides novel data. Furthermore, we focused on depression scores at discharge of the program, so we could consider spontaneous remission of depressive symptomatology, for instance as part of a short-term adjustment disorder [10]. Previous studies have also shown that especially patients whose depression scores do not improve in response to treatments, including cardiac rehabilitation, have a poorer prognosis than patients who were only depressed at the beginning of rehabilitation [9, 11]. Also, another study found that depressed CVD patients who did not respond to treatment with the antidepressant mirtazapine were at higher risk of experiencing a CVD-related event [21].

There are several limitations of our study that deserve mention. We included patients who were able to participate in a cardiac outpatient rehabilitation program. This might produce a selection bias because the severity of the CVD, the depression level and motivation might influence participation. Therefore, the study results cannot be transferred to other cardiac patients, including those with greater physical and mental impairment. Furthermore, we did not statistically control for further potential predictors, e.g. medication, physical activity, and adherence to therapy in order to avoid model overfitting. Controlling for such variables would have added strength to our study findings. In order to limit the burden on patients, we applied only one scale to measure depressive symptoms. Had we shown similar results with different measurements for depressive symptoms, this would have strengthened the validity of our results. As part of the comprehensive exercise-based cardiac rehabilitation program, patients participated in a stress management group.

No further systematic treatments that specifically reduce depression were provided. However, a meta-analysis by Rutledge et al. [8] confirmed that exercise-based cardiac rehabilitation treatments and mental health therapies (i.e., psychotherapies and antidepressants) showed similar effects on depression severity in patients with CHD. The present study is the first to investigate an association of depression with postrehabilitation outcomes in a sample of patients with three different primary diagnoses of CVD (i.e., CHD, chronic heart failure and aortic valve surgery). Studying this heterogeneous sample was justified because all patients completed the same rehabilitation program and only on top received information and therapeutic elements that were specifically tailored for each diagnosis of CVD. For further studies we recommend considering a control group as this might strengthen the significance of the findings.

In conclusion, depressive symptoms during rehabilitation predicted future CVD-related hospitalizations during a follow-up period of 3.5 years. We observed a dose-response relationship between depression scores and risk of a subsequent CVD-related event. Additionally, depressive symptom scores were reduced during rehabilitation. Therefore, cardiac rehabilitation programs might benefit patients' cardiovascular prognosis if an emphasis is put on reducing depressive symptoms during rehabilitation through effective means.

\section{Acknowledgments}

The authors wish to thank Tina Rohrbach and Viviane Guéra for performing telephone interviews and Annette Kocher for editorial assistance.

\section{Conflict of Interest}

The authors have no conflicts of interest to disclose.

References

Cardiology 2015;131:80-85
Meyer/Hugentobler/Stauber/Wilhelm/ Znoj/von Känel
1 Sans S, Kesteloot H, Kromhout D: The burden of cardiovascular diseases mortality in Europe. Eur Heart J 1997;18:1231-1248.

2 Willich SN, Müller-Nordhorn J, Kulig M, Binting S, Gohlke H, Hahmann H, et al: Cardiac risk factors, medication, and recurrent clinical events after acute coronary disease. Eur Heart J 2001;22:307-313.

3 Graham I, Atar D, Borch-Johnsen K, Boysen G, Burell G, Cifkova R, et al: European guidelines on cardiovascular disease prevention in clinical practice: executive summary. Eur Heart J 2007;28:2375-2414. DOI: $10.1159 / 000375231$ 
4 von Känel R: Psychosocial stress and cardiovascular risk - current opinion. Swiss Med Wkly 2012;142:w13502.

5 Bush DE, Ziegelstein RC, Patel UV, Thombs BD, Ford DE, Fauerbach JA, et al: Post-myocardial infarction depression. Evid Rep Technol Assess 2005;123:1-8.

6 Barth J, Schumacher MA, Herrmann-Lingen C: Depression as a risk factor for mortality in patients with coronary heart disease: a metaanalysis. Psychosom Med 2004;66:802-813.

7 van Melle JP, de Jonge P, Spijkerman TA, Tijssen JGP, Ormel J, van Veldhuisen DJ, et al: Prognostic association of depression following myocardial infarction with mortality and cardiovascular events: a meta-analysis. Psychosom Med 2004;66:814-822.

8 Rutledge T, Redwine SR, Linke SE, Mills PJ: A meta-analysis of mental health treatments and cardiac rehabilitation for improving clinical outcomes and depression among patients with coronary heart disease. Psychosom Med 2013;75:335-349.

9 Milani RV, Lavie CJ: Impact of cardiac rehabilitation on depression and its associated mortality. Am J Med 2007;120:799-806.

10 Albus C, Beutel ME, Deter H-C, Fritzsche K, Hellmich M, Jordan J, et al: A stepwise psychotherapy intervention for reducing risk in coronary artery disease (SPIRR-CAD) - rationale and design of a multicenter, random- ized trial in depressed patients with CAD. J Psychosom Res 2011;71:215-222.

11 Carney RM, Blumenthal JA, Freedland KE, Youngblood M, Veith RC, Burg MM, et al: Depression and late mortality after myocardial infarction in the Enhancing Recovery in Coronary Heart Disease (ENRICHD) study. Psychosom Med 2004;66:466-474.

12 Blum MR, Schmid J-P, Eser P, Saner H: Longterm results of a 12-week comprehensive ambulatory cardiac rehabilitation program. J Cardiopulm Rehabil Prev 2013;33:84-90.

13 Herrmann C, Buss U, Snaith RP: HADS-D: Hospital Anxiety and Depression Scale deutsche Version. Ein Fragebogen zur Erfassung von Angst und Depressivität in der somatischen Medizin. Bern, Huber, 1995.

14 Bjelland I, Dahl AA, Haug TT, Neckelmann D: The validity of the Hospital Anxiety and Depression Scale. An updated literature review. J Psychosom Res 2002;52:69-77.

15 Do CB, Batzoglou S: What is the expectation maximization algorithm? Nat Biotechnol 2008;26:897-899.

16 Babyak MA: What you see may not be what you get: a brief, non-technical introduction to overfitting in regression-type models. Psychosom Med 2004;66:411-421.

17 Snaith RB: The Hospital Anxiety and Depression Scale. Health Qual Life Outcomes 2003; 1:29-32.
18 Lichtman JH, Bigger JT, Blumenthal JA, Frasure-Smith N, Kaufmann PG, Lespérance $\mathrm{F}$, et al: Depression and coronary heart disease: recommendations for screening, referral, and treatment: a science advisory form the American Heart Association Prevention Committee of the Council on Cardiovascular Nursing, Council on Clinical Cardiology, Council on Epidemiology and Prevention, and Interdisciplinary Council on Quality of Care and Outcomes Research: endorsed by the American Psychiatric Association. Circulation 2008;118: 1768-1775.

19 Lavie CJ, Milani RV: Adverse psychological and coronary risk profiles in young patients with coronary artery disease and benefits of formal cardiac rehabilitation. Arch Intern Med 2006;166:1878-1883.

20 Lespérance F, Frasure-Smith N, Talajic M, Bourassa MG: Five-year risk of cardiac mortality in relation to initial severity and oneyear changes in depression symptoms after myocardial infarction. Circulation 2002;105: 1049-1053.

21 de Jonge P, Honig A, van Melle JP, Schene AH, Kuyper AMG, Tulner D, et al: Nonresponse to treatment for depression following myocardial infarction: association with subsequent cardiac events. Am J Psychiatry 2007; 164:1371-1378. 